\title{
Using LTE-Sim in New Hanover Decision Algorithm for 2-Tier Macrocell-Femtocell LTE Network
}

\author{
Umar Danjuma Maiwada \\ Department of Computer-Science \\ Umaru Musa Yaradua University, \\ Katsina, Nigeria \\ Email: umar.danjuma [AT] umyu.edu.ng
}

\author{
Aminu Aminu Muazu \\ Department of Computer-Science \\ Umaru Musa YaraduaUniversity, \\ Katsina, Nigeria \\ Email:aminu.aminu [AT] umyu.edu.ng
}

\author{
Izaddeen Kabir Yakasai \\ Department of Information Tech. \\ MiddlesexUniversity, London \\ Email: 17h0892 [AT] ubd.edu.bn
}

Abstract --- Deployment of mini macrocell base stations can also be referred to as femtocells improve quality of service of indoor and outdoor users. Nevertheless, mobility management remains a key is sue with regards to their deployment. This paper is leaned towards this issue, with in-depth focus on the most important aspect of mobility management - handover. In handover management, making a handover decision in the LTE two-tier macrocell femtocell network is a crucial research area. Decision algorithms in this research, are classified and comparatively analyzed according to received signal strength, user equipment speed, cost function and interference. However, it was observed that most of the discussed decision algorithms fail to consider cell selection with hybrid access policy in a single macrocell multiple femtocell scenario, another observation was a majority of these algorithms lack the incorporation of us er equipment residence parameter. Not including this parameter boosts the number of unnecessary handover occurrence. To deal with these issues, a sophisticated handover decision algorithm is proposed. The proposed algorithm considers the us er's velocity, received signal strength, residence time as well as the femtocell base s tation's access policy. Simulation results have s hown that the proposed algorithm reduces the number of unnecessary handovers when compared to conventional received signal strength based handover decision algorithm.

Keywords--- User-equipment, Radio Signal Service, Long Term Evolution, Mobility Management, Handoff

\section{INTRODUCTION}

W ith the continuous rise in the number of mobile application users worldwide, mobile operators face huge demands for mobile data services. This has brought about a number of technological innovations that serve as alternative to traditional broadband services. Nowadays, mobile devices are pretty much used to carry out all sorts of functions; from acces sing trending applications like Facebook and twitter to video streaming applications like YouTube all the way to voice over IP (VoIP) calls such as Viber, Whatsapp, Tango down to real time medical applications like Medscape and micro medex. According to global mobile data traffic forecast update, 2014-2019 (Cisco, 2015), "mobile data traffic will grow at a compound annual growth rate (CAGR) of 57 percent from 2014 to 2019 , reaching 24.3 Exabyte per month by 2019." However, these small cells have since proven not to be the optimal solution when it comes to Capital and Operational Expenditures (CAPEX \& OPEX) and power supply as discussed in (Jarvinen, 2009). Femtocell Base Station (FBS) was introduced as a cost effective way to address high demand as well as optimizing the CAPEX and OPEX.

FBSs are connected to the owner's ISP network by the means of a backhaul connection. Moreover, the owner is in charge of powering a FBS (Jarvinen, 2009), (Femto-Forum, 2012) and (Andrews et al., 2012). In March 2011, Chingwa Telecom $(\mathrm{CHT})$ announced that it would provide heavy data users with free Femtocell Base Stations (FBSs) in order to offload the growing mobile data traffic on its Macrocells (Wireless-federation, 2011). According to a res earch carried out by Transparency Market Research (James, 2015) into the femtocell market, the FBS is expected to reach a $£ 2.8 \mathrm{bn}$ market value with an impres sive CAGR of $37.1 \%$ from 2013 to the year 2019. Handover is the process of transferring user equipment from its serving base station to a target base station. Handover in this context can be between two macrocells, macrocell and femtocell or between two neighbouring femtocells. Handover involves several stages and handover decision is one of its key phases.

\section{PROBLEM DEFINITION}

Despite the fact that femtocell base stations are incomparably useful, how to make sure their performance blossoms as technology evolves by the day remain a concern as well as a booming research area. This paper focuses on handover decision for inbound mobility in the two-tier macrocell femtocell LTE network. To maintain a seamless connection between multiple cells either in low, medium or high mobility, handover has to be smooth, and a seamless 
handover requires the time it takes the connection to make or break to be put into consideration.

All measurement reports and control are carried out in the former phase while the latter controls decision and execution of the handover. There has been a lot of research towards improving the handover decision phase, yet challenges on how to reduce the probability of low service interruption for users that are in a low, medium and high mobility s tate never fails to dis appear. Several handover decis ion algorithms were studied and it was identified that most handover decision algorithms do not address multiple femtocell scenario. The other is sue identified is the absence of a solution to UE fast hop-on hop-off scenario. These two issues can delay handover process, which can lead to call dropping and QoS degradation.

\section{AIM \& OBJECTIVES OF THE WORK}

The aim of this research is to first of all investigate and conduct a survey of handover decision algorithms based on several decision parameters, and propose a new handover decision algorithmbased on the investigated schemes. Some of the parameters investigated are Received Signal Strength (RSS), UE Velocity, UE membership, to mention but few. The objectives of the work are given below.

-To research the femtocell technology with special reference to managing UEmobility within the femtocell coverage area. -To review the past and previous work in the area of mobility management, with special reference to handover decision algorithms for inbound and outbound mobility in the two-tier macrocell femtocell network.

-To investigate and evaluate the most important parameter incorporation with regard to handover decision for UEs in femtocell coverage areas.

\section{LITERATURE REVIEW}

A lot of research has been going on in the area of handover decision for two-tier macrocell femtocell network. (Moon \& Cho, 2009) (Moon \& Cho, 2010) (Xu et al., 2011) (LopezPerez et al., 2010) (Lopez-Perez et al., 2010) (Jeong et al., 2011) (Ulvan et al., 2010) (Zhang et al., 2011) (Shih-Jung Wu, 2011) (Shaohong et al., 2009) (Zhang et al., 2010) (Xu et al., 2010) (Lee et al., 2010) (Reguiga et al., 2011) (Xenakis et al., 2012) (Yang et al., 2011) (Chowdhury et al., 2009) (Kim \& Lee, 2010) (Becvar \& P, 2010) (Xenakis et al., 2012). A significant number of decision algorithms have been respectively proposed. More than half of the proposed algorithms represent a mixture of the handover decision parameters discussed. The main motivation behind this categorization is related to the main concept of the original research as well as their result analysis and the main parameters that affect them. The parameter mixture in the consulted literature led to a categorization of handover decision algorithms into:

1. Received Signal Strength

2. UE Velocity
3. Cost function and Interference based

4. Energy Efficiency (EE) and Path Los s (PL)

5. Type of Traffic (TT)

6. Bandwidth availability

\section{METHODOLOGY}

In order to overcome the aforementioned challenges, a new handover decision algorithm is proposed. The algorithm focuses on the UE velocity, residence time, cell search methods, received signal quality and members hip status. The proposed algorithmaddresses two key issues, the first is the scenario where a UE enters the femtocell coverage and quickly exits due to medium or high mobility. This brings about two unnecessary handovers per femtocell coverage, inbound and outbound. To address this is sue, the algorithm relates the UE speed to a speed threshold, uses a fixed UE residence time parameter to determine how long the UEstays in the target femtocell coverage and compares the received signal strength to a set threshold. The other issue the algorithm aims to address is that of a scenario where a UE leaves serving macrocell coverage area for a femtocell but meets several femtocell base stations in the destination. The algorithm will addres s how the UEis going to selectits target cell. To carry out this task, the algorithm utilizes the proximity estimation feature to combine UEmembers hip and neighbor cell list.

\section{ALGORITHM AND EVALUATION}

\section{A. Proposedhandover decision algorithmand performance evaluation}

There are two identified is sues; one is that of handover decision in a heavily dense femtocell deployment scenario, the other is that of hop-on hop-off. Below is a discussion on this is sues and how the proposed algorithmaddresses them.

\section{A.1. Multiple Femtocell Scenarios}

Most handover decision algorithms are purposely designed for macrocell handover to single femtocell. This comes with several drawbacks, as femtocells are not just heavily deployed nowadays, but in addition, they are consumer deployed; hence we will be safe to say that the deployment of femtocell base stations in some degree, unplanned. Let's say for example we have a macrocell and a couple of thousand femtocell base stations within this macrocell coverage. A mobile UE will definitely find it hard to select its preferred target cell, hence signaling overhead delay and QoS degradation follows. The proposed algorithmaddresses this is sue by incorporating NCL and CSG.

Neighborcell list is a database of potential or candidate base stations that are in proximity to a cell. Each base station provides UEs connected to it with this list so that they can choose the best one during handover (Nguyen et al., 2010). NCL assists in easing the whole proces s of cell selection 
thereby improving the handover procedure and to a greater extent, userquality of experience.

\section{A..2 Fast hop---on hop---offscenario}

Frequent and redundant handover is one big problem for femtocell-deployed environment. Femtocell coverage area is typically small in size and there is a chance to stay for only a very short time whenever a UEthat is on high-speed mobility pas ses. Two unnecessary handovers are resulted froma high speed UE passing through a femtocell coverage, one is an unnecessary handover from macrocell to femtocell and the other is from femtocell back to the macrocell. Unnecessary handovers lead to frequent call drops, which in turn could result in poor user experience. Therefore, it is es sential to minimize the rate of frequent and unnecessary handovers. In order to address this issue, a parameter, $\mathrm{T}$ is introduced to determine how long the UE stays in the femtocell coverage before it compares the serving cell's RSS to that of the femtocell base station using cell admis sion control. We can see the hop-on hop-off scenario where the UEpasses through femtocell coverage and suddenly leaves it.

\section{B. Proposedalgorithm}

HOM or hysteresis is a variable that denotes the RSS threshold between serving and target cells. HOM guarantees the target cell is the most suitable cell the UEcamps on amid handover. A TTT quality is the time period that is needed for fulfilling HOM condition. Both HOM and TTT are utilized for decreasing redundant handovers, which is also known as "Ping-Pong effect". At the point when a UE is encountering this impact, handover occurs from a serving cell to a target cell and from the target cell back to the serving cell again in a little timeframe. This impact builds the overhead signaling resources, diminishes the overall throughput as well as overall sys temdelay brought about by buffering the inbound traffic at the target cell when every handover happens. Consequently, viably preventing redundant handovers is crucial. TTT limits the handover activity from being activated within a certain time span. A handover activity must be performed after the TTT condition has been fulfilled. At the point when a UE is moving far from the serving cell, the RSRP, which the UEgets from the serving cell, will decrease as time increments. In the meantime, the UE will move towards the target cell, in this manner the RSRP and the UE gets from the target cell will increment proportionally with time. The flowchart of the proposed algorithm depicted and kicks off after the UEs velocity as well as the RSS at both serving and target cells are measure. It is divided into three phases:

1. The serving cell is a femtocell.

2. The serving cell is not femtocell but UE belongs to some CSG.

3. The serving cell is not femtocell and UEdoesn't belong to any CSG but velocity Vexceeds a certain limit, Vth.

\section{6.3. Performanceevaluation}

1. Channel models: The simulator comes with two new indoor path loss models. The first path loss model is given by;

$\mathrm{p} ! \mathrm{dB}=127+30 \log$ !"

$\mathrm{R} 1000$

Where $\mathrm{R}$ is the distance between the UE and receiving cell measured in meters.

The second path loss model is given by;

$\mathrm{P}$ ! dB = A log!" $10+\mathrm{B}+\mathrm{C} \log \log$ !" $\mathrm{f} ! / 5+\mathrm{X}$

Where:

$\mathrm{R}$ is the distance between the UEand receiving cell measured in meters, $f c$ is the central frequency, and

$\mathrm{A}, \mathrm{B}, \mathrm{C}$ are the barriers between the UEand receiving cell.

The second path loss model is more complex than the first model. However, it has more accuracy level.

2. For the investigation of the algorithm's performance evaluation, Power-based algorithm is utilized, yet for its straight forwardness, two parameters are added to do the research work, and these are hysteresis and TTT. Adding parameters of HOM and TTT demands for modifications in the code of the LTE-Sim. To present HOM, the accompanying condition is adopted (Adding a HOM variable):

$($ RXpower $>$ targetRXpower $+\mathrm{HOM})$

For TTT, a counter related to each eNB is presented, that tallies the time where the past condition is ensured.

If (RXpower > targetRXpower+HOM) \{TTT_counter++;

If (TTT_counter $>=$ TTT_threshold) \{

targetRX $\overline{\text { power }}=\mathrm{RXpower}$;

targetNode $=$ probableNewTargetNode; \{\}

Another variable is presented (TTT_counter) for each eNB that keeps hint of the time where the condition (RXpower $>$ targetRXpower + HOM) is ensured. When this time surpasses the TTT threshold, a handover happens.

List of parameters used in carrying out the simulation -Bandwidth 20MHz, Microcell power 40dBm, Femtocell power 20dBm, number of buildings 2, Apartments per building 20, size of the apartment $100^{2}$, Microcell radius (m) 500 , Femtocell radius (m) 10, Number of totalUEs in macrocell coverage $30, \mathrm{UE}$ velocity $(\mathrm{km} / \mathrm{hr}) 1530$ and 120 , TTT (secs) 3, RSS thres hold 1 -70dBm, RSS threshold 2 $72 \mathrm{dBm}$, Active Factor 1. Please observe that the macrocell trans mis sion power exceeds that of femtocell base stations. The simulator has two mobility models; randomdirection and random walk. 


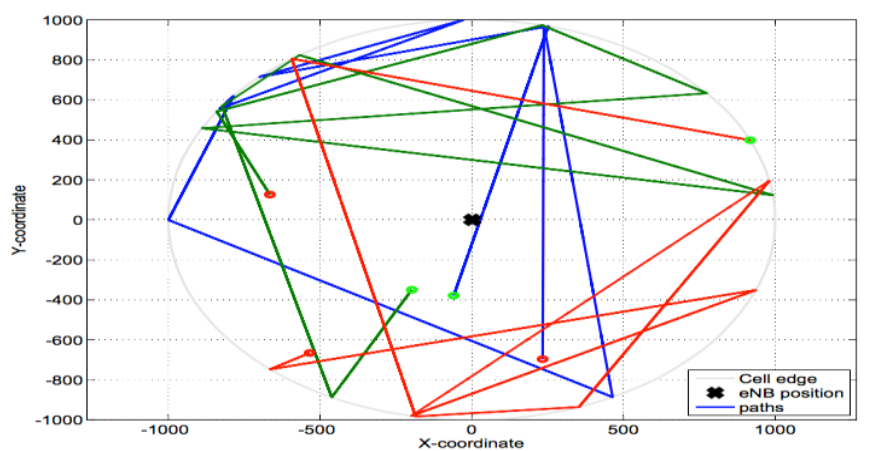

Figure 1: Example of randommobility pattern in LTE---Sim

LTE-Sim has two types of building configurations: dual stripe and $5 \times 5$ apartment grid. For this simulation, two dual stripes are used, the figure below shows building ty pe used in this performance evaluation. This is because we want to use $40 \mathrm{HeNBs}$ and $5 \times 5$ apartment grid consisting of only 25 apartments. (Piro et al., 2011) talks about further details with regards to this simulator.

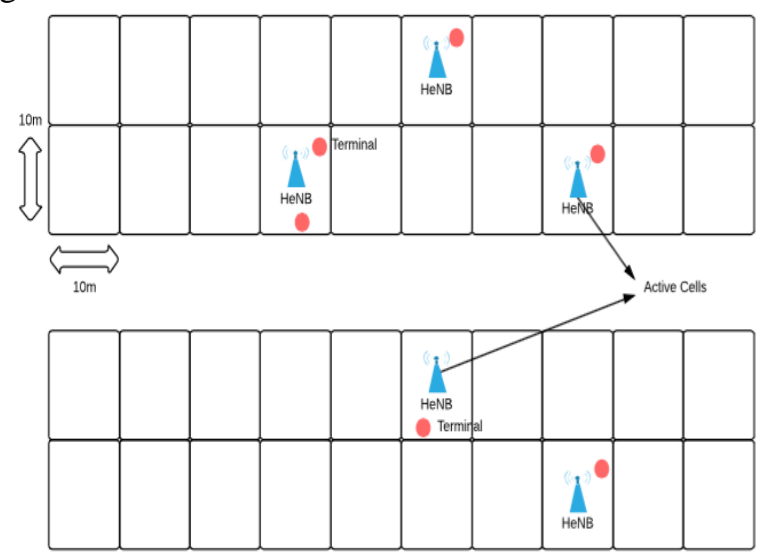

Figure 2: Two dual stripe Building topology

Setting active parameter to 1 means that all the femtocell base stations available in the building (i.e. $40 \mathrm{HeNBs}$ since there are 40 apartments and each apartment has $1 \mathrm{HeNB}$ ) are up and running. The use of femtocell CSG feature in aiding handover is included in the decision algorithmbut LTE-Sim doesn't have the CSG feature. To address this, we assume that for every $10 \mathrm{HeNBs}, 1$ is a non-CSG.

\section{C.1. Result andAnalysis}

The simulation begins with UEs moving in randomdirection within the macrocell coverage, which consists of multiple FABs. With a specific end goal to simulate the UE mobility, the simulator assigns to each cell and UE, a unique identifier. In addition, it initiates the UE's position as well as its configured velocity. Then it keeps track and records each UE's movement every $1 \mathrm{~ms}$. Specifically, it records the UEs Cartesian position and the unique identifier of the UEand the serving cell. In the simulator, whenever a handover occurs, the simulator communicates the recorded information to a new serving cell and deletes theold information.

For each simulation, result was obtained on the number of handover recorded in relation to the number of $\mathrm{UE}$ in mobility at that point in time. In addition, the default power based handover algorithm was simu lated and compared to the proposed algorithm.

This is because we assume the power based handover algorithm to be a representative of RSS based handover decision scheme.

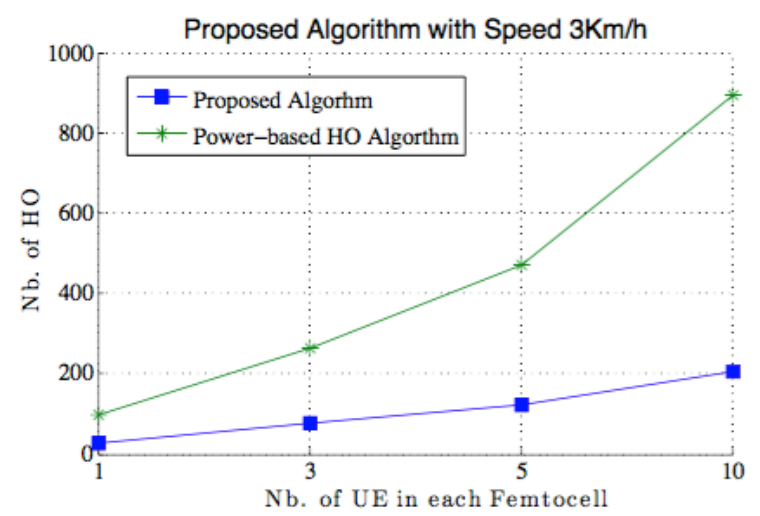

Figure 3: Proposed algorithm with velocity of $3 \mathrm{~km} / \mathrm{hr}$.

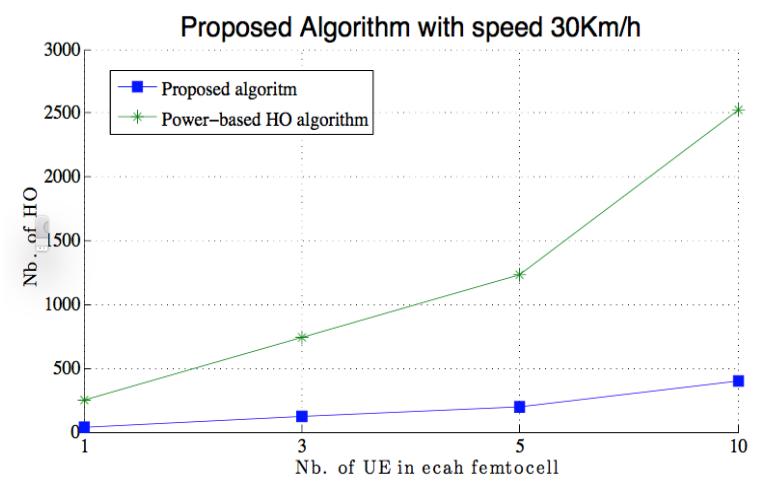

Figure 4: Proposed alg orithm with velocity of $30 \mathrm{~km} / \mathrm{hr}$.

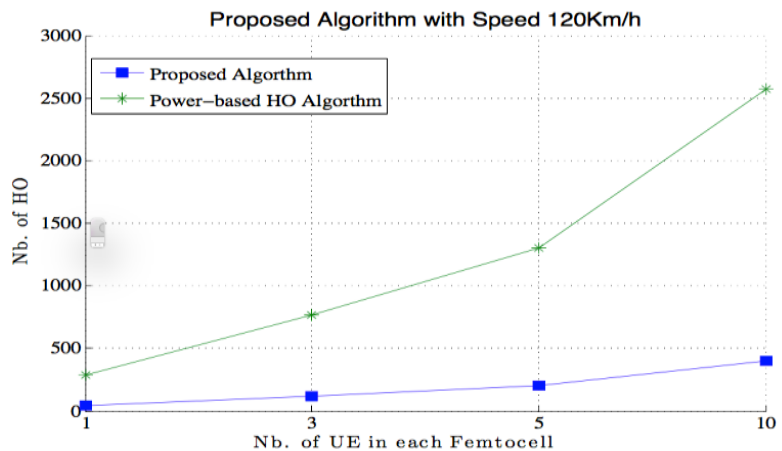

Figure 5: Proposed algorithm with velocity of $120 \mathrm{~km} / \mathrm{hr}$.

From the above results, we can say that the introduced time interval, $\mathrm{T}$ has a huge impact on the number of handover 
unnecessary handover. For the conventional power based handover decision algorithm, the number of unnecessary handover runs to thousands as the UEnumber increases, this happens because the mobile UE is moving fast and yet has handover to each and every femtocell base station it passes. Meaning that for each femtocell station passed, two unnecessary handovers occur. Comparing the proposed algorithm with the conventional power based algorithm, we can see that the number of unnecessary handovers has been significantly reduced. This is as a result of the time, $\mathrm{T}$ that the UE has to s tay for a minimum of before handover phase gets triggered. Likewise, result has shown that the proposed handover decision algorithm returns fewer unnecessary handover. It can als o be obs erved that the velocities in these cases are $3 \mathrm{~km} / \mathrm{hr}$ and $15 \mathrm{~km} / \mathrm{hr}$. respectively. The reas on for the reduction in unnecessary handover number here is because the UEresidence time of 3 seconds.

\section{CONCLUSION}

The growing demand for high-speed Internet connection has led to major technological advancements, including femtocell base stations. Femtocell base stations are small inexpensive low power base cellular base stations used for extending signals received from macrocells. The deployment of femtocell has since become a norm in modern day telecommunication industry. However, these deployments come with several challenges, including but not limited to mobility management. Handover has proved to be the most important property of mobility management for femtocell deployments in LTE networks. In addition, deciding when and where constitutes the critical part of a handover process. Nine different handover decision alg orithms were reviewed in this research, most of which were found to address a single macrocell single femtocell scenario. This scenario is not realistic in view if the heavy and unplanned deployment of femtocell in recent years. The main contribution of this research is the new decision algorithmproposed to solve the highlighted is sues above. The algorithmdeals with inbound, outbound and intra cell handovers of CSG and non-CSG member UEs. It also addresses the is sue of cell search using a combination of NCL and CSG. Finally, the proposed algorithm was evaluated by the means of simulation. However, not all segments were simulated; the introduction of a time to trigger of parameter of 3 seconds has proved to significantly reduce the number of redundant handovers for UEs in medium and high mobility.

\section{FUTURE WORK}

In femtocell networks, efficient mobility management is an integral component of providing seamless QoE for users. This res earch work focused on handover decision alg orithms with concentration in cell search u sing hybrid CSGas well as UE membership status using a TTT of 3 seconds. Even though performance evaluation was conducted, it is not extensive, hence the need for an extensive simulation. The research has mainly considered reduction of unnecessary handovers; however, it is advisable to further investigate the handover performance in terms of throughput, SINR, packet los s, delay, probability of handover occurrence and energy saving techniques in more complex scenarios. Handover procedure has other constituents like measurement control and report, handover execution. Furthermore, open is sues in mobility management such cell identification, access control, cell search; cell selection and res election need to be looked into in future works. Finally, femtocells on transport systems such as high-speed trains and busses will turn out to be more common in the near future. The need for reducing the burden on macrocells cannot be overemphasized, hence a future research direction.

\section{REFERENCES}

[1] A. Galindo-Serrano, L. Giupponi, M. Dohler, (2010) "Cognition and Docition in OFDM A Based Femtocell Networks," IEEE Global Telecommunications Conference, pp.1-6, Dec. 2010.

[2] Agilent technologies (John Wiley \& Sons), (2013). LTE and the Evolution to $4 \mathrm{G}$ Wireless: Design and Measurement Challenges.

[3] Alcatel-Lucent, (2009) Strategic White Paper, the LTE Network Architecture, a Comprehensive Tutorial, Cited at: xii, 8,10

[4] Haijun Zhang, Xiangming Wen, Bo Wang, Wei Zheng, Yong Sun (2010) "A Novel Handover Mechanism between Femtocell and Macrocell for LTE based Networks", Communication Software and Networks, ICCSN'10. Second Conference on, pp 228-231, Feb 2010.

[5] Hamza, J., (2009) Long Term Evolution (LTE) - A Tutorial, Network System Laboratory, Simon Fraser University, Canada, October 13, 2009. Cited at: 8

[6] Holma, H. and Toskala, A., (2009) LTE for UMTS OFDMA and SC-FDMA Based Radio Access, Wiley Publishers, 2009. Cited at: xii, 8, 9

[7] HuiZhou, Donglin Hu, Shiwen Mao, Prathima Agrawal, Saketh Anuma Reddy (2013) "Cell Association and Handover Management in Femtocell Networks", Wireles s Communications and Networking Conference (WCNC), 2013 IEEE Conference, pp 661-666, April 2013.

[8] Hussain, S., (2009) Dynamic Radio Resource Management in 3GPP LTE, Blekinge Tekniska H•ogskola, Karlskrona, Sweden, January 2009. Cited at: 8

[9] K.SethomBen Reguiga F. Mhiri, R. Bouallegue., (2011) "Handoff Management in Green FEMTOCELL Network", International Journal of Computer Applications (0975 - 8887) Volume 27- No.4, August 2011

[10] Mostafa Zaman Chowdhury and Yeong Min Jang (2013) "Handover management in high-dense femto cellular 
networks", EURASIP Journal on Wireless Communications and Networking, pp 1-21, December 2013, 2013:6.

[11] Rhode \& Schwarz, n.d.(2011) LTE--- Advanced(3GPP Rel.11) Technology Introduction WHITE PAPER. [Online] Available at: https://cdn.rohdeschwarz.com/pws/dl_downloads/dl_application/applicat ion_notes/1ma232/1MA2 32_1E_LTE_Rel11.pdf.

[12] Saketh Anuma Reddy, HuiZhou, Donglin Hu, Prathima Agrawal (2013) "Handoff Management with A Delay Requirement in Femtocell Networks", IEEE Journal, pp 119-123, March 2013.

[13] Saketh Anuma Reddy, (2014) Handover Management in Femtocell Networks May 4, 2014.

[14] Sesia, S. et al, (2011) LTE - The UMTS Long Term Evolution, From Theory to Practice, Second Edition, Wiley Publishers, 2011. Cited at: xii, 6, 7, 8, 10, 14, 15

[15] T. Zahir, K. Arshad, A. Nakata, K. Moessner, (2013) "Interference Management in Femtocells," in Common Surveys \& Tutorials, vol. 15, pp. 293-311, 2013.

[16] Webb, W., (2007). The future of wireless communications. John Wiley and Sons. Lxix 